\title{
Hungry Bone Syndrome after Parathyroidectomy for Primary Hyperthyroidism
}

\section{Giorgio Ghilardi* and Loredana De Pasquale ${ }^{2}$}

${ }^{1}$ Università degli Studi di Milano - Dipartimento di Scienze della Salute - Clinica Chirurgica Generale - via A. Di Rudini, 8 - Milano, Italy ${ }^{2}$ Ospedale San Paolo Polo Universitario - Dipartimento di Chirurgia - via A. Di Rudini, 8 - Milano, Italy

\begin{abstract}
Parathyroidectomy is the treatment of choice in patients with primary hyperparathyroidism (PHPT). This disease affects calcium metabolism at the level of bone tissue and renal tubules, resulting in hypercalcaemia, often asymptomatic, associated with hypophosphataemia and hypomagnesaemia.
\end{abstract}

Sudden suppression of parathyroid hormone (PTH), caused by successful parathyroidectomy, in patients with preoperative high levels of PTH and hypercalcaemia from enhanced bone turnover, may induce severe postoperative hypocalcaemia that may lead to symptoms of tetany. This relatively uncommon condition is known as "hungry bone syndrome" (HBS), because it is believed to be due mainly to enhanced bone formation.

Several risk factors have been advocated for HBS, and the syndrome is reported to be more likely to rise in subjects with severe preoperative bone disease. Other modifiable risk factors are preoperative vitamin D deficiency and high PTH and calcium levels.

Treatment of HBS is basically the administration of high amounts of calcium immediately after the onset of postoperative hypocalcaemia. Supplements of active metabolites of vitamin D, as well as magnesium in depleted subjects are complementary in supporting bone remineralization. Oral supplementation may be requested for months after parathyroidectomy.

Prevention is poorly documented, but it is reasonable to propose the correction of vitamin D deficit and the use of bisphosphonates aimed to lower PTH levels and bone resorption before parathyroidectomy.

Keywords: Primary hyperparathyroidism; Parathyroidectomy; Hungry bone syndrome; Hypocalcaemia; Hypercalcaemia

\section{Introduction}

Hungry Bone Syndrome (HBS) may occur in patients who have undergone Parathyroidectomy for Primary Hyperparathyroidism (PHPT), and is characterised by prolonged severe hypocalcaemia. Patients who preoperatively showed a large parathyroid adenoma, high preoperative serum calcium, serum Parathyroid Hormone (PTH) and serum alkaline phosphatase levels, skeletal manifestations as osteitis fibrosa cystica, brown tumours and severe osteoporosis, and old age are exposed to the risk of developing HBS [1].

In hyperparathyroidism, hypercalcaemia is mainly due to increased bone turnover with enhanced osteoclastic bone activity and increased reabsorption of calcium in the renal tubules. After parathyroidectomy, the PTH stimulus is abruptly removed. The excessive osteoclastic activity shuts down, and is replaced by an avid osteoblastic activity, resulting in rapid and marked increase in bone calcium uptake, predisposing the patient to symptomatic hypocalcaemia [2].

\section{Primary Hyperparathyroidism}

The hormone secreted by the four parathyroid glands, parathyroid hormone, is a cornerstone of calcium metabolism. Parathyroid chief cells receptors are activated by low levels of serum ionised calcium and secrete PTH. It induces calcium bone osteoclasts-mediated resorption and renal tubular calcium reabsorption. Intestinal calcium absorption is enhanced as well throw PTH induced hydroxylation of 25-OHvitamin $\mathrm{D} 3$ to the activated form 1,25-OH. By negative feed back, raised serum ionised calcium decreases $\mathrm{PTH}$ secretion to maintain adequate serum calcium levels [3].

Primary hyperparathyroidism (PHPT) is the commonest cause of hypercalcaemia, a condition that has become more frequently recorded because of popularization of routine screening tests that uncover PHPT well before symptoms appear [4].
Hypercalcaemia is defined as a raised serum total calcium level greater than $10.3 \mathrm{mg} / \mathrm{dL}(>2.58 \mathrm{mmol} / \mathrm{L})$; severe hypercalcaemia, serum total calcium level greater than $14 \mathrm{mg} / \mathrm{dL}(>3.5 \mathrm{mmol} / \mathrm{L})$, is associated with acute signs and symptoms. Mild hypercalcaemia is often asymptomatic.

Prevalence of PHPT in western countries is increasing as well [5] and ranges between $0.2 \%$ and $1 \%$, with a gender imbalance between males and females of 1:3. Although it can occur at any age, this disease affects people of over 55 at a rate up to $2 \%$ [6-8].

Sporadic PHPT is the commonest form and accounts for more than $85 \%$ of cases: it is supported by PT adenoma of the chief cells, affecting only one gland. PT hyperplasia accounts for less than $15 \%$ of cases and carcinoma is very rare (1\% or less) [9].

PHPT may also be a part of some familial disorders, namely Multiple Endocrine Neoplasia (MEN) type 1 and type 2A, familial hyperparathyroidism-jaw tumour syndrome, neonatal severe hyperparathyroidism, and familial isolated hyperparathyroidism.

In MEN 1 (Wermer syndrome) PT hyperplasia is associated to endocrine pancreas tumours and pituitary tumours (3Ps: parathyroid,

*Corresponding author: Giorgio Ghilardi, Università degli Studi di Milano Dipartimento di Scienze della Salute - Clinica Chirurgica Generale - via A. D Rudinì, 8 - Milano -Italy, Tel: 39-02-8137613; E-mail: giorgio.ghilardi@unimi.it

Received December 06, 2013; Accepted January 28, 2014; Published February 05, 2014

Citation: Ghilardi G, De Pasquale L (2014) Hungry Bone Syndrome after Parathyroidectomy for Primary Hyperthyroidism. Surgery Curr Res 4: 168. doi:10.4172/2161-1076.1000168

Copyright: $\odot 2014$ Ghilardi G, et al. This is an open-access article distributed under the terms of the Creative Commons Attribution License, which permits unrestricted use, distribution, and reproduction in any medium, provided the original author and source are credited. 
pancreas, pituitary). For the syndrome to be clinically manifest, the inherited germinal autosomal dominant mutation of the oncosuppressor gene MEN1, located on the chromosome 11, must be associated to somatic mutations. Other endocrine or cutaneous tumours may be present in up to $30 \%$ of the cases.

MEN 2 (Sipple syndrome) is characterized by medullary thyroid carcinoma and pheochromocytoma. The mutation of RET (REarranged during Transfection) proto-oncogene in the chromosome 10 is a genetic autosomal dominant disorder underlining this disease. PT hyperplasia or adenoma is involved in $10-20 \%$ and is the cause of hyperparathyroidism in MEN 2A. PT glands are not involved in MEN 2B [10].

Another familial condition, inherited as an autosomal dominant disease, is familial hypocalciuric hypercalcaemia. It is characterized by an inactivating mutation in the calcium sensing receptors gene, generating increased levels of serum calcium and reduced sensitivity of the kidney to these increased levels, with resulting relative hypocalciuria. Unsuppressed or elevated serum PTH and typically normal renal function are seen as well, while hypercalcaemic symptoms are generally absent [11-14].

\section{Parathyroidectomy}

Parathyroidectomy is the only cure for PHPT in current practice. The purpose of surgical treatment in PHP is to remove enough abnormal parathyroid tissue to reduce the long-term destructive effects on bone and the negative effects of high serum calcium levels and keep the patient normocalcaemic.

The possibility that two or more PTs are responsible of hyperparathyroidism is a major problem of PT surgery, because a reliable preoperative diagnosis is not yet available. In fact, either echography and sestamibi scintigram often fail to show whether or not there is a single gland responsible for the serum PTH increase.

Since the advent of intraoperative PTH (IOPTH) dosage, the treatment of choice was bilateral cervical exploration to identify all the four PTs: removal of "enlarged" gland was then accomplished on the basis of surgeon's judgement and reoperation for residual PHPT was frequent. Another risk of this extensive approach is postoperative permanent hypoparathyroidism secondary to excessive PT tissue removal [15]. Moreover, such an invasive approach increases the risk of injury to the laryngeal nerves. Intraoperative identification of the hyper-functioning PT gland(s) was performed then by radiolabeled sestamibi guided surgery: this technique is still particularly useful in redo operations. The background signal from other tissues and the rapid clearance of radiolabeled sestamibi prevented this method from gaining large acceptance in primary operations $[16,17]$.

IOPTH monitoring is to date the most accurate way to be sure that the hyper-functioning PT has been effectively removed, and, along with improved preoperative imaging techniques, made minimally invasive parathyroidectomy (MIP) the favoured treatment. MIP has been shown as successful as bilateral cervical exploration, but safer, because the risk of injury to the recurrent laryngeal nerves is lower. Additionally, MIP is much more tolerated by the patients and showed to be cost effective [18-22].

A successful parathyroidectomy results in a sudden decrease of PTH (due to its short half-life) that is intraoperative monitored. PTH half-life is independent of renal function but is increased by body mass index.

PTH is rapidly metabolized (less than 4 minutes) in the kidney and liver by enzymatic rupture in two fragments [23] a biologically active $\mathrm{N}$-terminal fraction and an inactive C-terminal fraction. Intact PTH assays are effective in measuring the kinetic of incretion of this hormone, because they measure either the hormone before degradation (1-84 aminoacids) and the $\mathrm{N}$-terminal fragment (7-84 aminoacids), while they do not cross-react with the inactive C-terminal fragment.

Three IOPTH criteria are most commonly used currently: all refer to a basal sample, taken after induction of anaesthesia and intubation but before skin incision, versus a second sample taken 10 or 15 minutes after gland removal.

The first criterion is the serum PTH value decrease of more than $50 \%$ from the highest pre-excision value (Miami criterion); drop of more than 50\% from pre-incision IOPTH level at 10 minutes (Vienna criterion); decrease of more than $50 \%$ and into the normal laboratory range after 15 minutes of excision (Halle criterion) [24-27].

\section{Hungry Bone Syndrome}

Hungry bone syndrome is a complication of parathyroid excision surgery, occurring when the sudden decrease in serum PTH is associated to the enhanced calcium uptake by the demineralized bone. The PTH stimulus on renal tubular reabsorption of calcium falls abruptly too. The net effect of such calcium metabolism inversion is hypocalcaemia, that may typically cause symptoms of tetany.

Table 1 resumes the clinical presentation of hyper- and hypocalcaemia.

Severe postparathyroidectomy hypocalcaemia has an estimated incidence of $12 \%$ (with an extremely wide range of 4 to $87 \%$ ), and may be as much severe and prolonged as to be life threatening and require urgent intensive therapy.

It is noticeable that the most frequent presentation of symptoms of mild tetany occurs with serum calcium levels still in the range of normality. Prolonged hypercalcaemia secondary to PHPT may down regulate calcium receptors and a condition of relative hypocalcaemia may occur soon after PT excision, causing tingling and numbness of fingers or lips.

The predictive value of preoperative laboratory investigations (namely serum calcium, PTH, alkaline phosphatase, albumin, magnesium) is questioned, but observations seem to have the tendency to link higher probability of HBS to major alterations of these indicators.

HBS is currently considered actual till postoperative hypocalcaemia recovers after parathyroidectomy, and calcium supplementation can be safely interrupted. This occurs when bone turnover turns back to

\begin{tabular}{|l|l|}
$\begin{array}{l}\text { Symptoms of severe } \\
\text { hypercalcaemia }\end{array}$ & Symptoms of severe acute hypocalcaemia \\
\hline Fatigue & Syncope \\
\hline Weakness & Angina \\
\hline Polydipsia & Congestive heart failure (reversible) \\
\hline Polyuria & Perioral and/or finger tingling and numbness \\
\hline Dehydration & Carpopedal spasm, Chvostek sign, Trusseau sign \\
\hline Nausea and/or vomiting & Muscular cramps (back and lower limbs) \\
\hline Abdominal pain & Wheezing (from bronchospasm) \\
\hline Constipation & Dysphagia \\
\hline Bone pain & Voice changes (spasm of the larynx) \\
\hline Mental status changes & Irritability, mental status changes \\
\hline & Seizures and/or uncontrolled movements \\
\hline
\end{tabular}

Table 1: Symptoms of severe hypercalcaemia and severe acute hypocalcaemia. 
normal and the function of the residual non-pathologic glands regains normal PTH secretion levels [28].

Within 3-4 days of successful parathyroidectomy, serum calcium levels may drop to $<2.1 \mathrm{mmol} / \mathrm{L}$, but patients with HBS show a progression in decrease of calcaemia well beyond this term. The degree of serum calcium level fall is a function of preoperative PTH level and related bone turnover. Serum phosphate decreases as well for the duration of the syndrome, and alkaline phosphatase may remain elevated for months, because of its involvement in bone formation. A marker of bone formation, serum osteocalcin, has reported to maintain high levels up to one year after surgery, in some cases of severe preoperative osteoporosis [29].

Recovery of skeletal manifestations of PHPT is the consequence of the cure, and increase in bone mineral density has been documented to reach the remarkable percentages of $35-131 \%$ at one year [30].

Bone formation can be demonstrated both by conventional radiographies and bone scintigraphy. Recovery of sub-periosteal resorption can be showed at follow-up radiograms as well as remineralization of brown tumours. Pathological fractures heal more rapidly and osteolytic lesions tend to disappear [30-32].

A typical feature of calcium uptake in restoring bone is the "flare phenomenon" at skeletal scintigram: previously ignored or underestimated osteoclastic lesions show high calcium uptake activity after curative parathyroidectomy, a feature similar to that occurring in treated bone metastasis where bone resorption areas turn on bone formation ones. Since bone scintigraphy is not a routine issue in parathyroidectomy follow up, the incidence of this phenomenon is unknown, but in studied patients flare-up was observed after months of surgery [33-35].

\section{How to Manage HBS}

Serum calcium, phosphate and PTH have a circadian rhythm in healthy people: peak phosphate and nadir calcium levels are recorded at 3.00 a.m. On the contrary, nadir phosphate and peak calcium levels are recorded at 3.00 p.m. [36]. PTH rises and falls twice a day (late afternoon-early evening and late evening-early morning), reaching nadir at mid-morning. Gender differences exist in that premenopausal women show less rapid but longer overnight rise than men [37,38]. This rhythm is lost in PHPT and re-conquered after parathyroidectomy. Serum calcium and phosphate seem to be the most effective factors involved in the maintenance of this rhythm, but other endogenous factors have a role, growth hormone and prolactin appearing as the main actors [39].

After parathyroidectomy for PHPT, calcium levels decrease suddenly, and this phenomenon may be excessively amplified by magnification of bone formation.

The management of HBS must satisfy enhanced calcium request either in emergency and long-term settings.

Since hypocalcaemia occurs suddenly after surgery, it must be monitored in the first few hours of operation. Repeated blood samples are needed to assess the tendency to lowering serum calcium levels, and serum phosphate and magnesium must be monitored as well.

Not uncommonly, tetany symptoms can occur with normal serum calcium levels, an expression of relative hypocalcaemia.

Functional hypoparathyroidism may be enhanced by serum magnesium depletion (e.g. diabetes, diuretics, magnesium wasting renal disorders, alcoholism) or excess (diet magnesium supplementation in subjects with chronic renal diseases, laxatives, antiacids). Moreover, magnesium may suppress PTH release by activation of extracellular calcium-sensing receptors, a calcium-like effect [40].

High levels of serum phosphate are known to stimulate gene transcription and PTH secretion independently of calcium and/or calcitriol levels [36].

Depletion of vitamin D is often associated to PHPT, and restoration of normal levels of $25(\mathrm{OH}) \mathrm{D}$ is to be attained, given the importance of this vitamin for calcium metabolism.

Oral supplementation of large amounts of calcium (up to 12 grams per day) is required to treat HBS; intravenous calcium gluconate is administered in the acute presentation, until recovery from symptoms of tetany has been gained and oral administration can maintain adequate levels of serum calcium.

Electrocardiographic monitoring is advisable when intravenous administration of large amounts of calcium-containing solutions is required, to prevent the risk of dysrhythmias that may occur in case of too rapid correction of hypocalcaemia [41].

When administering calcium orally, the different content of calcium of current preparations has to be taken in account, because there is a wide variability in elemental calcium percentages: calcium carbonate has the most favourable rate $(40 \%)$ followed by citrate $(20 \%)$. Other preparations require a too large number of tablets to reach adequate serum calcium levels, and patient compliance to therapy results negatively affected [40].

The concomitant administration of vitamin $\mathrm{D}$ (as active forms) is mandatory, to support the needs of bone remineralization.

Restoration of normal levels of phosphate usually follows the normalization of serum calcium, and magnesium must be supplemented only in cases of hypomagnesaemia. Magnesium deficiency may require high replacement at high doses that can be administered intravenously as sulphate; when oral administration is sufficient, magnesium oxide may be employed successfully. Hypocalcaemia cannot be definitely resolved until normal levels of serum magnesium are obtained [42].

On a long term perspective, calcium and active vitamin $\mathrm{D}$ metabolites supplementation is guided by serum levels of calcium and $25(\mathrm{OH})$ vitamin $\mathrm{D}$. Markers of bone turnover are useful to determine the metabolic condition of the skeleton: its normalization is an indicator of stable resolution of HBS.

The conquest of normality in calcium metabolism and bone turnover may require several months and it is not uncommon for patients to need calcium and vitamin D supplementation for as long as one year.

\section{Options to Prevent HBS}

Since vitamin D depletion is detected in as much as $76 \%$ patients with PHPT [43], it has been considered a risk factor for developing postoperative hypocalcaemia and HBS $[44,45]$.

Preoperative vitamin D supplementation in depleted patients resulted in decreased levels of PTH, better control of hypercalcaemia and bone turnover [46]. Although this practice seems to be justified and advisable, no randomized controlled study evaluated the impact of preoperative vitamin $\mathrm{D}$ restoring therapy on postoperative hypercalcaemia in PHPT.

Bone resorption is actually best managed with bisphosphonates. 
These drugs are currently used in bone disorders associated with calcium mobilization for their osteoclastic inhibition and remineralization activity. The use of bisphosphonates to prevent HBS has been advocated, but their beneficial effects may only develop on a long-term basis and the normalization of alkaline phosphatase should be achieved before surgery is attempted.

Short-term bisphosphonate therapy, in fact, may reduce bone resorption, but decrease of bone formation needs a longer time to reduce calcium request: thus, hypocalcaemia may result severely enhanced after parathyroidectomy.

Although the theory is convincing, preoperative long-term administration of bisphosphonates to prevent HBS has no longer been tested in randomized controlled trials, and data from several limited series are conflicting [47-50].

\section{Conclusion}

In mild as well in severe PHPT parathyroidectomy allows to normalize either serum calcium and PTH. Bone resorption decreases and mineral density increases in patients successfully operated.

Minimally invasive parathyroidectomy is as effective as extended neck exploration for PT previously localized but largely safer in preserving laryngeal nerve function and integrity.

IOPTH monitoring is essential in the modern approach to parathyroidectomy.

HBS may occur as a complication of parathyroidectomy in patients whose PTH and serum calcium levels are very elevated preoperatively.

The incidence of HBS appears to decrease in time, possibly due to early diagnosis of hypercalcaemia and PHPT. Better management of bone resorption and appropriate preoperative treatment contributed to minimize the incidence of HBS.

Prevention of HBS with activated vitamin D in depleted subjects and bisphosphonates, in a long-term setting, is advisable, although evidence for the latter comes only from small uncontrolled series. Short-term bisphosphonates administration seems to be less useful and, in some instances, revealed to be a possible trigger to HBS.

Treatment of severe HBS after parathyroidectomy requires intravenous calcium administration under electrocardiographic monitoring, high dose activated vitamin $\mathrm{D}$, intravenous magnesium sulphate or oral magnesium oxide in the case of deficiency.

Mild HBS may be managed with oral administration of large amounts of calcium, looking out for the mineral content of the preparation given.

\section{Declaration of Interest}

The authors declare that there is no conflict of interest that could be perceived as prejudicing the impartiality of the research reported.

\section{References}

1. Brasier AR, Nussbaum SR (1988) Hungry bone syndrome: clinical and biochemical predictors of its occurrence after parathyroid surgery. Am J Med 84: $654-660$

2. Smith D, Murray BF, McDermott E, O'Shea D, McKenna MJ, et al. (2005) Hungry bones without hypocalcaemia following parathyroidectomy. J Bone Miner Metab 23: 514-515.

3. Khan AA, Bilezikian JP, Potts JT Jr; Guest Editors for the Third International Workshop on Asymptomatic Primary Hyperparathyroidism (2009) The diagnosis and management of asymptomatic primary hyperparathyroidism revisited. J Clin Endocrinol Metab 94: 333-334.
4. Gurrado A, Piccinni G, Lissidini G, Di Fronzo P, Vittore F, et al. (2012) Hypercalcaemic crisis due to primary hyperparathyroidism - a systematic literature review and case report. Endokrynol Pol 63: 494-502.

5. Quiros RM, Valentin C, DeCresce R, Prinz RA (2003) Intraoperative total serum calcium levels, unlike intraoperative intact PTH levels, do not correlate with cure of hyperparathyroidism. J Surg Res 114: 57-63.

6. Eigelberger MS, Clark $\mathrm{OH}$ (2000) Surgical approaches to primary hyperparathyroidism. Endocrinol Metab Clin North Am 29: 479-502.

7. Cobin RH, Gharib H, Bergman DA (2001) AACE/AAES medical/surgical guidelines for clinical practice: management of thyroid carcinoma. American Association of Clinical Endocrinologists. American College of Endocrinology. Endocr Pract 7: 202-220

8. Adami S, Marcocci C, Gatti D (2002) Epidemiology of primary hyperparathyroidism in Europe. J Bone Miner Res 17 Suppl 2: N18-23.

9. Khan A, Bilezikian J (2000) Primary hyperparathyroidism: pathophysiology and impact on bone. CMAJ 163: 184-187.

10. Camanni F, Ghigo E Eds (2011) Malattie del sistema endocrino e de metabolismo - IV ed. Edi.Ermes, Milano.

11. Eastell R, Arnold A, Brandi ML, Brown EM, D'Amour P, et al. (2009) Diagnosis of asymptomatic primary hyperparathyroidism: proceedings of the third international workshop. J Clin Endocrinol Metab 94: 340-350.

12. Tfelt-Hansen J, Brown EM (2005) The calcium-sensing receptor in norma physiology and pathophysiology: a review. Crit Rev Clin Lab Sci 42: 35-70.

13. Pallan S, Rahman MO, Kahn AA (2013) Diagnosis and treatment of primary hyperparathyroidism. BMJ 344:e1013

14. Christensen SE, Nissen PH, Vestergaard P, Mosekilde L (2011) Familia hypocalciuric hypercalcaemia: a review. Curr Opin Endocrinol Diabetes Obes 18: $359-370$.

15. Leiker AJ, Yen TW, Eastwood DC, Doffek KM, Szabo A, et al. (2013) Factors that influence parathyroid hormone half-life: determining if new intraoperative criteria are needed. JAMA Surg 148: 602-606.

16. Jaskowiak NT, Sugg SL, Helke J, Koka MR, Kaplan EL (2002) Pitfalls of intraoperative quick parathyroid hormone monitoring and gamma probe localization in surgery for primary hyperparathyroidism. Arch Surg 137: 659668.

17. Prescott JD, Udelsman R (2009) Remedial operation for primary hyperparathyroidism. World J Surg 33: 2324-2334.

18. Udelsman R, Donovan PI, Sokoll LJ (2000) One hundred consecutive minimally invasive parathyroid explorations. Ann Surg 232: 331-339.

19. Dillavou ED, Cohn HE (2003) Minimally invasive parathyroidectomy: 101 consecutive cases from a single surgeon. J Am Coll Surg 197: 1-7.

20. Udelsman R, Donovan PI (2004) Open minimally invasive parathyroid surgery World J Surg 28: 1224-1226.

21. Mazzaglia PJ, Berber E, Kovach A, Milas M, Esselstyn C, et al. (2008) The changing presentation of hyperparathyroidism over 3 decades. Arch Surg 143: 260-266.

22. Mihai R, Barczynski M, lacobone M, Sitges-Serra A (2009) Surgical strategy for sporadic primary hyperparathyroidism: an evidence-based approach to surgical strategy, patient selection, surgical access, and reoperations. Langenbecks Arch Surg 394: 785-798.

23. Romani AM, Panarese A, La Torre V (2007) The role of intraoperative parathyroid hormone assay in the surgical management of hyperparathyroidism. Annal Ital Chir 78: 91-96.

24. Agarwal G, Barakate MS, Robinson B, Wilkinson M, Barraclough B, et al. (2001) Intraoperative quick parathyroid hormone versus same-day parathyroid hormone testing for minimally invasive parathyroidectomy: a cost-effectiveness study. Surgery 130: 963-970.

25. Carneiro DM, Solorzano CC, Nader MC, Ramirez M, Irvin GL 3rd (2003) Comparison of intraoperative iPTH assay (QPTH) criteria in guiding parathyroidectomy: which criterion is the most accurate? Surgery 134: 973979.

26. Chiu B, Sturgeon C, Angelos P (2006) Which intraoperative parathyroid hormone assay criterion best predicts operative success? A study of 352 consecutive patients. Arch Surg 141: 483-487. 
Citation: Ghilardi G, De Pasquale L (2014) Hungry Bone Syndrome after Parathyroidectomy for Primary Hyperthyroidism. Surgery Curr Res 4: 168. doi:10.4172/2161-1076.1000168

Page 5 of 5

27. Riss P, Kaczirek K, Heinz G, Bieglmayer C, Niederle B (2007) A "defined baseline" in PTH monitoring increases surgical success in patients with multiple gland disease. Surgery 142: 398-404.

28. Witteveen JE, van Thiel S, Romijn JA, Hamdy NA (2013) Hungry bone syndrome: still a challenge in the post-operative management of primary hyperparathyroidism: a systematic review of the literature. Eur $\mathrm{J}$ Endocrinol 168: R45-53.

29. Agarwal G, Mishra SK, Kar DK, Singh AK, Arya V, et al. (2002) Recovery pattern of patients with osteitis fibrosa cystica in primary hyperparathyroidism after successful parathyroidectomy. Surgery 132: 1075-1083.

30. Yong TY, Li JY (2010) Mediastinal parathyroid carcinoma presenting with severe skeletal manifestations. J Bone Miner Metab 28: 591-594.

31. França TC, Griz L, Pinho J, Diniz ET, Andrade LD, et al. (2011) Bisphosphonates can reduce bone hunger after parathyroidectomy in patients with primary hyperparathyroidism and osteitis fibrosa cystica. Rev Bras Reumatol 51: 131137.

32. Rathi MS, Ajjan R, Orme SM (2008) A case of parathyroid carcinoma with severe hungry bone syndrome and review of literature. Exp Clin Endocrino Diabetes 116: 487-490.

33. Ajmi S, Sfar R, Trimeche S, Ben Ali K, Nouira M (2010) Scintigraphic findings in hungry bone syndrome following parathyroidectomy. Rev Esp Med Nucl 29 $81-83$.

34. Hardoff R, Frajewicki V (1996) Bone scintigraphy in hungry bone syndrome following parathyroidectomy. J Nucl Med 37: 1371-1373.

35. Kuzucu A, Soysal O, Savli H (2002) Giant mediastinal parathyroid adenoma presenting with a hyperparathyroid crisis and leading to postoperative hungry bone syndrome. Eur J Surg 168: 747-749.

36. de Francisco AL, Cobo MA, Setien MA, Rodrigo E, Fresnedo GF, et al. (1998) Effect of serum phosphate on parathyroid hormone secretion during hemodialysis. Kidney Int 54: 2140-2145.

37. Micozkadioglu H, Ozelsancak R, Yildiz I, Erken E, Zumrutdal A, et al. (2013) Circadian rhythm of serum phosphate, calcium and parathyroid hormone levels in hemodialysis patients. Clin Lab 59: 79-84.

38. Calvo MS, Eastell R, Offord KP, Bergstralh EJ, Burritt MF (1991) Circadian variation in ionized calcium and intact parathyroid hormone: evidence for sex differences in calcium homeostasis. J Clin Endocrinol Metab 72: 69-76.
39. Fraser WD, Ahmad AM, Vora JP (2004) The physiology of the circadian rhythm of parathyroid hormone and its potential as a treatment for osteoporosis. Curr Opin Nephrol Hypertens 13: 437-444.

40. Shoback D (2008) Clinical practice. Hypoparathyroidism. N Engl J Med 359 391-403.

41. Cooper MS, Gittoes NJ (2008) Diagnosis and management of hypocalcaemia BMJ 336: 1298-1302.

42. Natsui K, Tanaka K, Suda M, Yasoda A, Shigeno C, et al. (1996) Oxyphil parathyroid adenoma associated with primary hyperparathyroidism and marked post-operative hungry bone syndrome. Intern Med 35: 545-549.

43. Norenstedt S, Pernow Y, Brismar K, Sääf M, Ekip A, et al. (2013) Primary hyperparathyroidism and metabolic risk factors, impact of parathyroidectomy and vitamin $D$ supplementation, and results of a randomized double-blind study. Eur J Endocrinol 169: 795-804.

44. Silverberg SJ (2007) Vitamin D deficiency and primary hyperparathyroidism. J Bone Miner Res 22 Suppl 2: V100-104.

45. Bollerslev J, Marcocci C, Sosa M, Nordenström J, Bouillon R, et al. (2011) Current evidence for recommendation of surgery, medical treatment and vitamin $\mathrm{D}$ repletion in mild primary hyperparathyroidism. Eur J Endocrinol 165 851-864.

46. Rolighed L, Bollerslev J, Mosekilde L (2011) Vitamin D treatment in primary hyperparathyroidism. Curr Drug Saf 6: 100-107.

47. Kumar A, Ralston SH (1996) Bisphosphonates prevent the hungry bone syndrome. Nephron 74: 729 .

48. Lee IT, Sheu WH, Tu ST, Kuo SW, Pei D (2006) Bisphosphonate pretreatment attenuates hungry bone syndrome postoperatively in subjects with primary hyperparathyroidism. J Bone Miner Metab 24: 255-258.

49. Gurevich Y, Poretsky L (2008) Possible prevention of hungry bone syndrome following parathyroidectomy by preoperative use of pamidronate. Otolaryngol Head Neck Surg 138: 403-404.

50. Corsello SM, Paragliola RM, Locantore $\mathrm{P}$, Ingraudo F, Ricciato MP, et al. (2010) Post-surgery severe hypocalcemia in primary hyperparathyroidism preoperatively treated with zoledronic acid. Hormones (Athens) 9: 338-342. 